\title{
Corticosteroids for community-acquired pneumonia: a critical view of the evidence
}

\author{
James D. Chalmers \\ Affiliation: Scottish Centre for Respiratory Research, University of Dundee, Dundee, UK. \\ Correspondence: James D. Chalmers, Scottish Centre for Respiratory Research, University of Dundee, \\ Dundee, DD1 9SY, UK. E-mail: j.chalmersadundee.ac.uk
}

0

@ERSpublications

The current evidence raises more questions than answers, and does not support routine use of corticosteroids for CAP http://ow.ly/t01g302QDhG

Community acquired pneumonia (CAP) remains a leading cause of morbidity and mortality, with mortality rates between $5 \%$ and $15 \%$ in hospitalised patients, rising to up to $30 \%$ in patients admitted to the intensive care unit (ICU) [1-3].

There has been little progress in the management of CAP since the widespread introduction of antibiotics in the 1950s, with no new classes of drugs licensed specifically for CAP and little progress in reducing mortality. The focus of international guidelines for CAP have been on "doing the simple things well", since early antibiotic administration, appropriate fluid resuscitation, early mobilisation and guideline-concordant antibiotics have been associated with improved outcomes, albeit with low-quality evidence [3-5].

Some of the lack of progress reflects the fact that, in many cases, mortality from CAP may be unavoidable. A third of patients admitted to hospital have "do not attempt resuscitation" orders, while only a quarter of patients dying from CAP receive mechanical ventilation before death, indicating a very high frequency of treatment restrictions $[6,7]$.

Nevertheless, there is a group of patients the CAP, usually with acute respiratory failure and septic shock, who die or experience prolonged and disabling hospital admissions despite antibiotic treatment and appropriate quality of care. These patients have marked systemic and pulmonary inflammation, providing the rationale for anti-inflammatory therapy.

This background is essential to understanding the current debate around the use of oral or intravenous corticosteroid treatment in CAP.

Two recent randomised controlled trials (RCTs), using very different steroid preparations and studying different patient populations, reported positive results in $2015[8,9]$. These were followed by a series of meta-analyses all suggesting positive data in favour of corticosteroid use [10].

In this issue of the European Respiratory Journal, WIRZ et al. [8] report an important subanalysis of their previous RCT of prednisolone $50 \mathrm{mg} v s$ placebo [11]. In the original study, 802 patients were randomised and steroid treatment was associated with a significant reduction in time to clinical stability (TCS) (1.4 days; hazard ratio 1.33, 95\% CI 1.15-1.50) [8]. Hospital discharge was 1 day shorter (6 versus 7 days, $\mathrm{p}=0.01$ ) with no difference in ICU admission or mortality. There was an increase in adverse events (OR $1.77,95 \%$ CI 1.24-2.52; $\mathrm{p}=0.002$ ) largely driven by an increase in hyperglycaemia [8].

This subanalysis attempted to address the key question of which patients may benefit and which may be harmed by treatment. Intriguingly, they identify that patients with pneumococcal pneumonia did not derive a benefit from steroids, with no improvement in the TCS or duration of antibiotic treatment [8]. This study also addresses concern that steroids might be harmful in the context of viral pneumonia. Patients with confirmed viral infection benefitted with a shortened TCS as with the overall group.

Received: July 052016 | Accepted: July 062016

Conflict of interest: Disclosures can be found alongside this article at erj.ersjournals.com

Copyright OERS 2016 
Nevertheless, the finding of lack of efficacy in the pneumococcal subgroup, the largest and most important microbiological subgroup in CAP, adds further question marks in an already confused picture [8].

In the other recently published RCT, TORREs et al. [9] performed a multicentre RCT in patients with severe CAP and a C-reactive protein (CRP) level $>150 \mathrm{mg} \cdot \mathrm{L}^{-1}$ on admission. Severe CAP was defined as a Pneumonia Severity Index (PSI) of V or the Infectious Diseases Society of America/American Thoracic Society criteria [1]. The study was conducted over 8 years, and randomised 61 patients to methylprednisolone and 59 patients to placebo for 5 days. The primary outcome was treatment failure, defined as a composite of development of shock, need for mechanical ventilation, death or radiographic progression [9]. The study showed a significant reduction in treatment failure of $18 \%$. This was driven largely by a $14 \%$ reduction in radiographic progression. Reductions in other end-points were not statistically significant. Length of stay and TCS were not affected by steroid treatment [9].

In 2011, MeIjvis et al. [12] compared 4 days of intravenous dexamethasone to placebo in hospitalised CAP patients and showed a 1-day reduction in the length of stay, with no improvements in ICU admission rate or mortality. The patient population had mild-moderate CAP according to the PSI. Hyperglycaemia was increased as an adverse event.

A meta-analysis by Simieniuk et al. [10] pooled these various studies, demonstrating a reduction in mortality that did not achieve statistical significance from 12 trials (rate ratio 0.67, 95\% CI 0.45-1.01), with significant reductions in mechanical ventilation and development of acute respiratory distress syndrome from five and four trials, respectively. While this might be considered encouraging, the possible mortality benefit was largely driven by a single controversial trial in which the mortality rate in the steroid arm was $0 \%$ [13]. The quality of evidence was predominantly low.

Nevertheless, in the face of a series of positive trials, leading voices advocate the routine use of corticosteroids for CAP [14]. So why should we be cautious?

First, there are well conducted RCTs showing no impact of steroid treatment. A trial conducted in the Netherlands randomised 213 patients to prednisolone $40 \mathrm{mg}$ for 7 days or placebo, with a primary outcome of clinical cure at day 7 [15]. This study found no benefit of steroid treatment and, importantly, found a trend toward fewer patients with cure at day $30(\mathrm{p}=0.08)$ with an increase in patients meeting the criteria for late failure, defined as a worsening or increase in symptoms after 7 days (OR 2.36, 95\% CI 1.05-5.31). Mortality and TCS were not different between the two groups [15].

Second, it is also important to look critically at the positive trials. The BLum et al. [11] and MeIJvis et al. [12] studies were criticised because the end-points may have been influenced by the antipyretic and anti-inflammatory effects of corticosteroids. Both SNIJDERS et al. [15] and MeIjvis et al. [12] demonstrated clearly that corticosteroids have an antipyretic effect, with resolution of fever $\left(>37.8^{\circ} \mathrm{C}\right)$ reached approximately 1 day earlier with steroids than placebo $[13,15]$. While this may be regarded as a benefit, it casts doubt on whether TCS is the right end-point to evaluate corticosteroids. TCS is a US Food and Drug Administration-approved end-point for antibiotic trials in CAP because TCS correlates well with future mortality and can therefore be used as a valid surrogate of response [16, 17]. Crucially, antibiotic treatment does not directly interfere with any of the criteria but steroids might. Fever may be artificially suppressed by corticosteroids. While studies have attempted to address this issue, the concern remains that this is a flawed end-point to test steroids [18]. Length of hospital stay is also partly dependent on defervescence and TCS. In the MeIjvis et al. [12] study, consistent reduction in CRP was part of the criteria for hospital discharge [12]. Steroids have been consistently shown to accelerate decline in CRP [12].

The Torres et al. [9] trial had significant methodological strengths in targeting severe CAP and using CRP to attempt to target patients with greater systemic inflammation. Nevertheless, limitations of the trial have been highlighted. The treatment failure outcome was largely driven by steroids preventing radiological progression, an outcome that is poorly characterised [9]. The study was not prospectively registered on a clinical trial database and the retrospective registration listed a different primary outcome (rate of nonresponsive to empirical therapy) to that ultimately reported in the paper (www.clinicaltrials.gov identifier number NCT00908713). In addition, although the study tried to enrich for severe CAP, 24-30\% were in PSI groups I-III and the mortality rate was only 3\% [9].

A further criticism of all of the steroid studies is that levels of improvement seen with steroids may be achieved with simple improvements in care. A reduction in length of stay from 7.5 to 6.5 days sounds valuable but, as in the MeIJvis et al. [12] study, in a population of patients in which $>50 \%$ of patients had PSI scores I-III, where inpatient care is often not required [19], this represents a surprisingly long baseline length of stay. Improvements of $>1$ day can be achieved with early mobilisation and good-quality inpatient care without new pharmacotherapies [4]. Likewise, reductions in duration of antibiotic therapy and intravenous antibiotic therapy can be achieved with simple clinical interventions [20, 21]. 
So, it is important to sound a note of caution. The quality of evidence currently available for corticosteroids would be unlikely to survive careful consideration by a clinical practice guideline panel. The recent positive trials increase the likelihood that steroids will find a role in the management of CAP but in my view, the data do not yet support routine use.

Additional RCTs will report soon and should shed additional light on the debate (NCT02517489 and NCT01743755). There is likely to be a subgroup of patients with CAP who derive significant benefit from corticosteroid treatment, but how to identify this group should be the focus of new research. Even in diseases with well-accepted roles for corticosteroids such as COPD and asthma, biomarkers can now identify groups of patients with steroid responsive and non-steroid responsive disease [22]. In a disease as heterogeneous as CAP, such studies will be difficult, but are essential.

Major improvements in length of stay, duration of antibiotic therapy and even mortality, greater than those demonstrated with corticosteroids, can be achieved through compliance with high-quality care recommendations, and yet audits show persistently poor compliance with guidelines [1-5]. Doing the simple things well should continue to take priority over the use of corticosteroids, for now.

\section{References}

1 Salih W, Schembri S, Chalmers JD. Simplification of the IDSA/ATS criteria for severe CAP using meta-analysis and observational data. Eur Respir J 2014; 43: 842-851.

2 Amaro R, Liapikou A, Cilloniz C, et al. Predictive and prognostic factors in patients with blood-culture-positive community-acquired pneumococcal pneumonia. Eur Respir J 2016; 48: 797-807.

3 Lim HF, Phua J, Mukhopadhyay A, et al. IDSA/ATS minor criteria aid pre-intensive care unit resuscitation in severe community-acquired pneumonia. Eur Respir J 2014; 43: 852-862.

4 Carratala J, Garcia-Vidal C, Ortega L, et al. Effect of a 3-step critical pathway to reduce duration of intravenous antibiotic therapy and length of stay in community-acquired pneumonia: a randomized controlled trial. Arch Intern Med 2012; 172: 922-928.

5 Garin N, Genne D, Carballo S, et al. $\beta$-lactam monotherapy vs $\beta$-lactam-macrolide combination treatment in moderately severe community-acquired pneumonia: a randomized non-inferiority trial. JAMA Intern Med 2014; 174: 1894-1901.

6 Chalmers JD, Taylor J, Mandal P, et al. Validation of the IDSA/ATS minor criteria for ICU admission in community-acquired pneumonia patients without major criteria or contraindications to ICU care. Clin Infect Dis 2011; 53: 503-511.

7 Kolditz M, Bauer TT, Konig T, et al. 3-day mortality in hospitalised community-acquired pneumonia: frequency and risk factors. Eur Respir J 2016; 47: 1572-1574.

8 Wirz S, Blum C, Schutz P, et al. Pathogen and antibiotic-specific effects of prednisolone in community-acquired pneumonia. Eur Respir J 2016; 48: 1150-1159.

9 Torres A, Sibila O, Ferrer M, et al. Effect of corticosteroids on treatment failure among hospitalised patients with severe community-acquired pneumonia and high inflammatory response: a randomized clinical trial. JAMA 2015; 313: 677-686.

10 Siemieniuk RA, Meade MO, Alonso-Coello P, et al. Corticosteroid therapy for patients hospitalized with community-acquired pneumonia: a systematic review and meta-analysis. Ann Intern Med 2015; 163: 519-528.

11 Blum CA, Nigro N, Briel M, et al. Adjunct prednisone therapy for patients with community-acquired pneumonia: a multicentre, double-blind, randomised, placebo-controlled trial. Lancet 2015; 385: 1511-1158.

12 Meijvis SC, Hardeman $\mathrm{H}$, Remmelts $\mathrm{HH}$, et al. Dexamethasone and length of hospital stay in patients with community-acquired pneumonia: a radnomised double blind placebo-controlled trial. Lancet 2011; 377: 2023-2030.

13 Confalonieri M, Urbino R, Potena A, et al. Hydrocorticosone infusion for severe community-acquired pneumonia: a preliminary randomized study. Am J Respir Crit Care Med 2005; 171: 242-248.

14 Restrepo MI, Anzueto A, Torres A. Corticosteroids for severe community-acquired pneumonia: time to change clinical practice. Ann Intern Med 2015; 163: 560-561.

15 Snijders D, Daniels JM, De Graaff CS, et al. Efficacy of corticosteroids in community-acquired pneumonia: a randomized double-blind clinical trial. Am J Respir Crit Care Med 2010; 181: 975-982.

16 Akram AR, Chalmers JD, Taylor JK, et al. An evaluation of clinical stability criteria to predict hospital course in community-acquired pneumonia. Clin Microbiol Infect 2013; 19: 1174-1180.

17 Aliberti S, Zanaboni AM, Wiemkin T, et al. Criteria for clinical stability in hospitalised patients with community-acquired pneumonia. Eur Respir J 2013; 42: 742-749.

18 Vestjens SM, Spoorenberg SM, Rijkers GT, et al. Antipyretic effect of dexamethasone in community-acquired pneumonia. Eur Respir J 2015; 46: 570-573.

19 Chalmers JD, Akram AR, Hill AT. Increasing outpatient treatment of mild community-acquired pneumonia: systematic review and meta-analysis. Eur Respir J 2011; 37: 858-864.

20 Ramirez JA, Srinath L, Ahkee S, et al. Early switch from intravenous to oral cephalosporins in the treatment of hospitalized patients with community-acquired pneumonia. Arch Intern Med 1995; 155: 1273-1276.

21 Murray C, Shaw A, Lloyd M, et al. A multidisciplinary intervention to reduce antibiotic duration in lower respiratory tract infections. J Antimicrob Chemother 2014; 69: 515-518.

22 Barnes NC, Sharma R, Lettis S, et al. Blood eosinophils as a marker of response to inhaled corticosteroids in COPD. Eur Respir J 2016; 47: 1374-1382. 